(C) 2005 The Japan Society of Applied Physics

\title{
Monte Carlo Simulation of Optical Properties of Phosphor-Screened Ultraviolet Light in a White Light-Emitting Device
}

\author{
Chien C. Chang*, Ruey-Lin Chern, C. Chung Chang, Chin-Chou Chu, Jim Y. Chi ${ }^{1}$, \\ Jung-Chieh $\mathrm{Su}^{1}$, I-Min $\mathrm{CHAN}^{1}$ and Jih-Fu Trevor WANG ${ }^{1}$ \\ Institute of Applied Mechanics, National Taiwan University, Taipei 106, Taiwan, Republic of China \\ ${ }^{1}$ Opto-Electronics and Systems Laboratories, Industrial Technology Research Institute, Hsinchu 310, Taiwan, Republic of China
}

(Received May 11, 2005; accepted June 2, 2005; published August 5, 2005)

In this paper, we study the optical properties of phosphor-screened ultraviolet light emitted by a quantum well through a chamber. The chamber contains randomly distributed red, blue and green phosphors, and is top-covered with a layer of omnidirectional photonic bandgap material. A Monte Carlo ray tracing method is developed to model the absorption, reflection and transmission for the excited radiation of the ultraviolet light as well as the visible light by the individual phosphor particles. The efficiency of emitting white light by synthesizing the visible light through the top substrate is investigated with respect to the weight ratio, the size of phosphor particles, the dimension of the chamber and the reflectivity of the side wall and the bottom substrate. [DOI: 10.1143/JJAP.44.6056]

KEYWORDS: Monte Carlo method, white light-emitting diode, photonic bandgap materials, conversion efficiency, ultraviolet light source, reflecting wall

\section{Introduction}

It has gained an increasing interest to synthesize white light from a light-emitting diode (LED). In particular, white LEDs have practical applications such as full-color flatpanel displays when combined with color filters, and incandescent bulbs or even fluorescent lamps as alternative lighting sources or equipments. These applications are of great importance due to small size, long lifetime, and the lower energy consumption of LEDs. In the past years, there are intensive efforts devoted to the study of the transfer efficiency of white light from other light sources. Basically, white light can be obtained by mixing different colors with adequate intensities. The most commonly used method is to combine a phosphor wavelength converter with an excitation source, such as blue light with yellow phosphors, ${ }^{1-4)}$ and ultraviolet light with blue, green and red phosphors. ${ }^{5,6)}$ Another method to achieve white LEDs is to use quantum-well structures which are able to emit red, green and blue light.7)

In the earlier study, we proposed a new design of white LEDs $^{8)}$ as shown in Fig. 1. The device consists of three parts: LED chip, LED chamber, and LED superstrate. On bottom of the chamber, ultraviolet light are emitted from an InGaN-based quantum well LED chip. The LED chamber contains a mixture of epoxy and red phosphor $\mathrm{La}_{2} \mathrm{O}_{2} \mathrm{~S}: \mathrm{Eu}$, green phosphor $\mathrm{ZnS}: \mathrm{Cu}, \mathrm{Al}$, and blue phosphor ( $\mathrm{Sr}, \mathrm{Ca}$, $\mathrm{Ba}, \mathrm{Mg})_{10}\left(\mathrm{PO}_{4}\right)_{6} \mathrm{Cl}_{2}$ :Eu used to re-emit visible light. The side wall of the chamber and the bottom substrate excluding the LED chip are considered to be totally or partially reflecting. On the top of the chamber, a layer of omnidirectional photonic bandgap material ${ }^{9)}$ is used to totally reflect the ultraviolet light and allow only passage of visible light. The efficiency of emitting white light by synthesizing the visible light through the top substrate depends on the number, size, shape, roughness, and optical properties of the phosphors such as index of refraction, absorption coefficient, quantum efficiency, as well as the optical properties of the matrix material and dimension of the chamber.

*E-mail address: changcc@gauss.iam.ntu.edu.tw

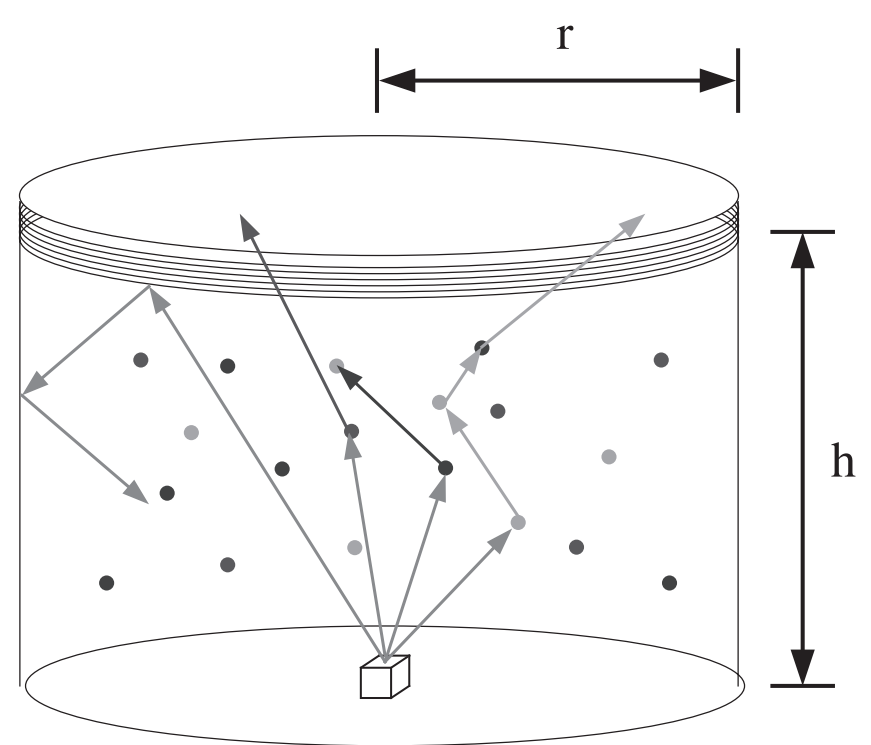

Fig. 1. Schematic of the white light-emitting diode with $r=1.2 \mathrm{~mm}$, $h=1.5 \mathrm{~mm}$. The ultraviolet light are emitted from the quantum well on the bottom substrate.

The phosphor particles considered here have a radius ranging from 2 to $6 \mu \mathrm{m}$. These are substantially larger than the wavelengths of the visible light, and therefore visible light as well as ultraviolet light can be modelled as rays of photons, each of which carries a photon energy with itself. By tracing a large number of rays of photons, the optical properties of phosphor-screened light can be obtained by counting the number and directions of the photons at the outlet of the chamber. Ray tracing includes free travelling of light, collisions of light photons with phosphors (reflection, transmission, absorption: emission and dissipation), reflection of light by the side wall and bottom substrate, as well as total reflection of ultraviolet light by the top superstrate. All the optical processes are required to follow Snell's law for plane waves incident on a flat surface, while the orientation of each collisional process, scattering direction of emitted light, and the chances of light to be reflected, refracted, 
transmitted or absorbed, emitted or dissipated are determined by sampling a set of appropriate random numbers.

In previous studies, Monte Carlo ray tracing methods have been developed for calculating the destinations and intensities of luminescent emission in a phosphor screen, ${ }^{10}$ ) modelling the optical properties of fluorescent powders, ${ }^{11)}$ and investigating the optical emission properties of a fluorescent display. ${ }^{12}$ ) Unlike these studies which deal with phosphors with fixed positions, the presently proposed Monte Carlo ray tracing method is mainly developed to simulate a randomly distributed phosphor particles, which means that the size, location, density, and even the shape of the phosphors can be arbitrarily distributed.

\section{Monte Carlo Method}

As mentioned in the previous section, the phosphor size ranges from 2 to $6 \mu \mathrm{m}$, which are much larger than the wavelengths of visible light. Therefore, the Monte Carlo ray tracing method is qualified for the simulation. Each ray carrying a photon energy undergoes a sequence of fundamental processes, which are repeated until the photon comes out of the chamber or is absorbed by a phosphor. The Monte Carlo ray tracing method is implemented in the following steps:

(1) Emission of the ultraviolet photons. First, we consider an ultraviolet photon emitted from bottom of the chamber according to the directivity pattern $\sin \theta_{i}$, where $\theta_{i}$ is the polar angle measured from the $z$-axis. By choosing a random number $Z_{1}$ between 0 and 1 as the probability of directivity $P_{\mathrm{d}}$, the polar angle of the emitted ultraviolet photon is determined by $\theta_{i}=$ $\sin ^{-1} P_{\mathrm{d}}$. The azimuthal angle $\phi_{i}$ is determined by a random number $Z_{2}$ between 0 and $2 \pi$.

(2) Collision on the phosphors. Initially, the photon is randomly directed, and after travelling a distance of a mean free path, the photon hits the surface of a phosphor particle. The mean free path $l_{\text {mean }}$ is determined as

$$
l_{\text {mean }}=\frac{V}{N_{\mathrm{p}} \sigma},
$$

where $V$ is the chamber volume, $N_{\mathrm{p}}$ is the number of the particles, and $\sigma=\pi r_{\mathrm{p}}^{2}$ is the cross section of the particle with radius $r_{\mathrm{p}}$.

(3) Orientation of the phosphors. Figure 2 shows the schematic diagram for the orientation of the phosphor
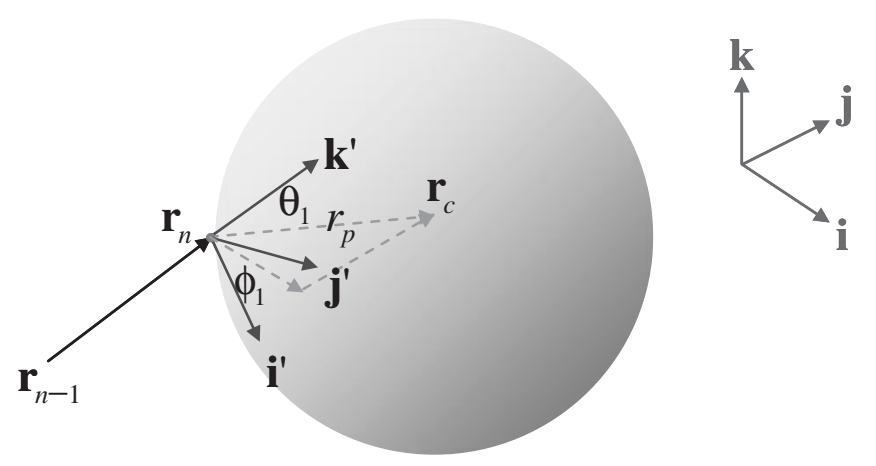

Fig. 2. Schematic of the orientation of the phosphor particle upon collision by the photon.

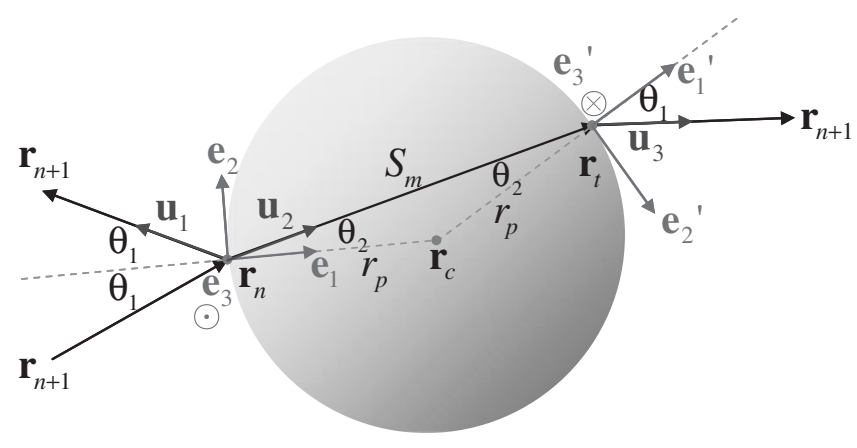

Fig. 3. Schematic of the reflection, refraction and transmission of the photon from the phosphor particle.

particle upon collision by the photon. Let $\mathbf{i}, \mathbf{j}, \mathbf{k}$ refer to the standard base vectors of a fixed coordinate system. In order to determine the location of the phosphor center, we define a local coordinate system with unit vectors

$$
\mathbf{k}^{\prime}=\frac{\mathbf{r}_{n}-\mathbf{r}_{n-1}}{\left|\mathbf{r}_{n}-\mathbf{r}_{n-1}\right|}, \quad \mathbf{j}^{\prime}=\frac{\mathbf{k}^{\prime} \times \mathbf{i}}{\left|\mathbf{k}^{\prime} \times \mathbf{i}\right|}, \quad \mathbf{i}^{\prime}=\mathbf{j}^{\prime} \times \mathbf{k}^{\prime},
$$

where $\mathbf{r}_{n}$ is the position vector of the photon at step $n$, and also the point of collision on phosphor. By choosing a random number $Z_{3}$ between $-\pi / 2$ to $\pi / 2$ as the incident angle $\theta_{1}$ between the incident photon direction and the inward normal of the phosphor particle at the point of incidence, and a random number $Z_{4}$ between 0 to $2 \pi$ as the azimuthal angle $\phi_{1}$ about the normal direction, the center of the sphere $\mathbf{r}_{\mathrm{c}}$ is given as

$$
\begin{aligned}
\mathbf{r}_{\mathrm{c}}= & \mathbf{r}_{n}+r_{\mathrm{p}}\left(\mathbf{i}^{\prime} \sin \theta_{1} \cos \phi_{1}\right. \\
& \left.+\mathbf{j}^{\prime} \sin \theta_{1} \sin \phi_{1}+\mathbf{k}^{\prime} \cos \theta_{1}\right) .
\end{aligned}
$$

(4) Construction of the incident plane. Figure 3 shows the schematic diagram for the reflection, refraction and transmission of the photon from the phosphor particle. In order to construct the incident plane on which the reflection, refraction or transmission occur, we define a local coordinate system with unit vectors

$$
\mathbf{e}_{1}=\frac{\mathbf{r}_{\mathrm{c}}-\mathbf{r}_{n}}{\left|\mathbf{r}_{\mathrm{c}}-\mathbf{r}_{n}\right|}, \quad \mathbf{e}_{3}=\frac{\mathbf{e}_{1} \times \mathbf{k}^{\prime}}{\left|\mathbf{e}_{1} \times \mathbf{k}^{\prime}\right|}, \quad \mathbf{e}_{2}=\mathbf{e}_{3} \times \mathbf{e}_{1} .
$$

Then, the direction of reflection is given as

$$
\mathbf{u}_{1}=-\mathbf{e}_{1} \cos \theta_{1}+\mathbf{e}_{2} \sin \theta_{1},
$$

and the direction of refraction as

$$
\mathbf{u}_{2}=\mathbf{e}_{1} \cos \theta_{2}+\mathbf{e}_{2} \sin \theta_{2} .
$$

(5) Reflection of the photons. Let $n_{\mathrm{e}}$ and $n_{\mathrm{p}}$ denote the refractive indices of the epoxy and phosphors, respectively. The average intensity of reflection for unpolarized light is given as $R_{\mathrm{S}}=\left(R_{\mathrm{s}}^{\mathrm{E}}+R_{\mathrm{s}}^{\mathrm{H}}\right) / 2$, where

$$
\begin{aligned}
& R_{\mathrm{s}}^{\mathrm{E}}=\left(\frac{n_{\mathrm{p}} \cos \theta_{2}-n_{\mathrm{e}} \cos \theta_{1}}{n_{\mathrm{p}} \cos \theta_{2}+n_{\mathrm{e}} \cos \theta_{1}}\right)^{2}, \\
& R_{\mathrm{s}}^{\mathrm{H}}=\left(\frac{n_{\mathrm{p}} \cos \theta_{1}-n_{\mathrm{e}} \cos \theta_{2}}{n_{\mathrm{p}} \cos \theta_{1}+n_{\mathrm{e}} \cos \theta_{2}}\right)^{2}
\end{aligned}
$$

are reflection coefficients for $\mathrm{E}$ and $\mathrm{H}$ polarizations, respectively, and the angle of refraction $\theta_{2}$ is then 
determined from Snell's law:

$$
n_{\mathrm{e}} \sin \theta_{1}=n_{\mathrm{p}} \sin \theta_{2} \text {. }
$$

By choosing a random number $Z_{5}$ between 0 and 1 as the probability of reflection, a photon is reflected if $Z_{5} \leq R_{\mathrm{s}}$, or refracted if $Z_{5}>R_{\mathrm{s}}$. If the photon is reflected, it travels with another mean free path, and hits another phosphor particle according to the same process described above.

(6) Refraction of the photons. If the photon is refracted, then there are two possibilities: transmitted or absorbed. According to the Beer-Bouguer-Lambert's exponential law of attenuation, ${ }^{13}$ ) the transmitted distance $S$ associated with a transmission probability $P_{\mathrm{t}}$ is given by

$$
P_{\mathrm{t}}=e^{-S k_{\mathrm{p}}},
$$

where $k_{\mathrm{p}}$ is the absorption coefficient of the particle. By sampling a random number $Z_{6}$ between 0 and 1 as the probability of transmission, the transmitted distance $S$ is given by

$$
S=\frac{1}{k_{\mathrm{p}}} \ln \frac{1}{P_{\mathrm{t}}} .
$$

The photon is transmitted through the phosphor if $S$ is larger than the maximum possible distance $S_{\mathrm{m}}=$ $2 r_{\mathrm{p}} \cos \theta_{2}$ of the refracted light inside the particle, and is absorbed otherwise.

(7) Transmission of the photons. In order to obtain the transmission direction, we construct a local coordinate system with unit vectors

$\mathbf{e}_{1}^{\prime}=\frac{\mathbf{r}_{\mathrm{t}}-\mathbf{r}_{\mathrm{c}}}{\left|\mathbf{r}_{\mathrm{t}}-\mathbf{r}_{\mathrm{c}}\right|}, \quad \mathbf{e}_{3}^{\prime}=\frac{\mathbf{e}_{1}^{\prime} \times \mathbf{u}_{2}}{\left|\mathbf{e}_{1}^{\prime} \times \mathbf{u}_{2}\right|}, \quad \mathbf{e}_{2}^{\prime}=\mathbf{e}_{3}^{\prime} \times \mathbf{e}_{1}^{\prime}$,

where $\mathbf{r}_{\mathrm{t}}=\mathbf{r}_{n}+S_{\mathrm{m}} \mathbf{u}_{2}$ is the position vector of the transmitted point. According to Snell's law, the direction of the transmission photon is given as

$$
\mathbf{u}_{3}=\mathbf{e}_{1}^{\prime} \cos \theta_{1}+\mathbf{e}_{2}^{\prime} \sin \theta_{1} .
$$

If the photon is transmitted, the travelling and collisional processes are repeated.

(8) Absorption of the photons. If the photon is absorbed, it is either re-emitted as a visible light and transmitted out of the phosphor particle, or dissipated into heat. By choosing a random number $Z_{7}$ between 0 and 1 as the probability of emission, the ultraviolet photon is emitted if $Z_{7}$ is smaller than the overall quantum efficiency $\eta_{\mathrm{Q}}$, or dissipated otherwise. The overall quantum efficiency is equal to the sum of internal and external quantum efficiencies, and here we use the value 0.674 , which is obtained from experiments. If the photon is already a visible one, then it is dissipated into heat.

(9) Emission of the photons. In the emission process, the ultraviolet photon is converted to a visible one, and reemitted in a random direction out of the particle. The emission direction can be determined by the polar angle $\theta_{\mathrm{e}}$ by sampling a random number $Z_{8}$ between 0 and $\pi$, and the azimuthal angle $\phi_{\mathrm{e}}$ by sampling a random number $Z_{9}$ between 0 and $2 \pi$. The emission point is then given as
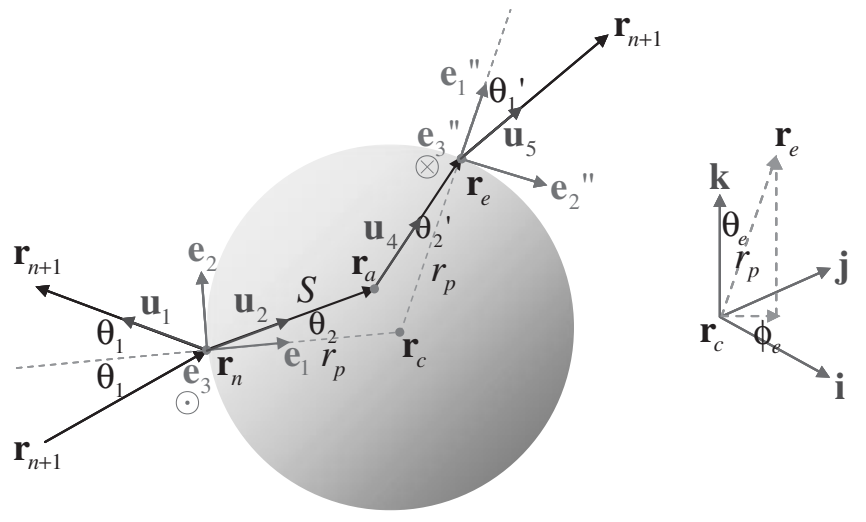

Fig. 4. Schematic of the absorption and emission of the photon by the phosphor particle.

$$
\mathbf{r}_{\mathrm{e}}=\mathbf{r}_{\mathrm{c}}+r_{\mathrm{p}}\left(\sin \theta_{\mathrm{e}} \cos \phi_{\mathrm{e}} \mathbf{i}+\sin \theta_{\mathrm{e}} \sin \phi_{\mathrm{e}} \mathbf{j}+\cos \theta_{\mathrm{e}} \mathbf{k}\right)
$$

Figure 4 shows the schematic diagram for the absorption and emission of the photon by the phosphor particle. The direction of emission is determined by

$$
\mathbf{u}_{4}=\frac{\mathbf{r}_{\mathrm{e}}-\mathbf{r}_{\mathrm{a}}}{\left|\mathbf{r}_{\mathrm{e}}-\mathbf{r}_{\mathrm{a}}\right|}
$$

where $\mathbf{r}_{\mathrm{a}}=\mathbf{r}_{n}+S \mathbf{u}_{2}$ is the position vector of the absorption point. Construct a local coordinate system with unit vectors

$$
\mathbf{e}_{1}^{\prime \prime}=\frac{\mathbf{r}_{\mathrm{e}}-\mathbf{r}_{\mathrm{c}}}{\left|\mathbf{r}_{\mathrm{e}}-\mathbf{r}_{\mathrm{c}}\right|}, \quad \mathbf{e}_{3}^{\prime \prime}=\frac{\mathbf{e}_{1}^{\prime \prime} \times \mathbf{u}_{4}}{\left|\mathbf{e}_{1}^{\prime \prime} \times \mathbf{u}_{4}\right|}, \quad \mathbf{e}_{2}^{\prime \prime}=\mathbf{e}_{3}^{\prime \prime} \times \mathbf{e}_{1}^{\prime \prime} .
$$

The angle between the emission photon direction and the outward normal $\theta_{2}^{\prime}$ is then given by

$$
\theta_{2}^{\prime}=\cos ^{-1}\left(\mathbf{u}_{4} \cdot \mathbf{e}_{1}^{\prime \prime}\right) \text {. }
$$

The direction of transmission of the re-emitted photon is given as

$$
\mathbf{u}_{5}=\mathbf{e}_{1}^{\prime \prime} \cos \theta_{1}^{\prime}+\mathbf{e}_{2}^{\prime \prime} \sin \theta_{1}^{\prime},
$$

where, according to Snell's law,

$$
\theta_{1}^{\prime}=\sin ^{-1}\left(n_{\mathrm{p}} \sin \theta_{2}^{\prime} / n_{\mathrm{e}}\right) .
$$

The color of the visible photon is determined by choosing a random number $Z_{10}$ between 0 and 3 . Red, blue or yellow color is assigned to the re-emitted photon if the integer part of $Z_{10}$ is equal to 0,1 , or 2 , respectively.

In the method described above, we have totally ten random numbers in the Monte Carlo simulation, which are summarized in the following:

$Z_{1}$ : sampled to determine the initial polar angle $\theta_{\mathrm{i}}$ of the photon;

$Z_{2}$ : sampled to determine the initial azimuthal angle $\phi_{\mathrm{i}}$ of the photon;

$Z_{3}$ : sampled to determine the incident angle $\theta_{1}$ of the photon upon collision with the phosphor;

$Z_{4}$ : sampled to determine the azimuthal angle $\phi_{1}$ of the photon upon collision with the phosphor;

$Z_{5}$ : sampled to determine the reflection of the photon from the phosphor;

$Z_{6}$ : sampled to determine the transmission distance through the phosphor; 
$Z_{7}$ : sampled to determine the emission of the photon from the phosphor;

$Z_{8}$ : sampled to determine the polar angle $\theta_{\mathrm{e}}$ for the reemitted photon;

$Z_{9}$ : sampled to determine the azimuthal angle $\phi_{\mathrm{e}}$ for the reemitted photon;

$Z_{10}$ : sampled to determine the color for the re-emitted photon.

\section{Results and Discussion}

A large number $\left(10^{4}\right)$ of ultraviolet photons are emitted in random directions from the bottom center of the chamber. The number of escaped visible photons from top of the chamber is counted, and the ratio of the number of visible photons to that of ultraviolet photons is the conversion efficiency $E_{\mathrm{c}}$ of the light-emitting device. First of all, we consider the side wall and the bottom substrate to be perfectly reflecting.

Figure 5 shows the conversion efficiency $E_{\mathrm{c}}$ of ultraviolet photons to visible photons for different weight ratios of phosphor particles with radius from 2 to $6 \mu \mathrm{m}$ with and without a top layer. In the case with a top layer, the conversion efficiency $E_{\mathrm{c}}$ is seen to reach a maximum $65 \%$ at the weight ratio $w=6 \%$, but changes little over the range from 4 to $7 \%$. Below $4 \%$ the conversion efficiency $E_{\mathrm{c}}$ decreases sharply with decreasing the weight ratio, while above $w=7 \%$ the conversion efficiency $E_{\mathrm{c}}$ also decreases quite rapidly with further increasing the weight ratio. At lower weight ratios, ultraviolet light photons have relatively little chance to be screened by the phosphors, while at large weight ratios, phosphor-screened photons have little chance to escape the chamber to reach the top layer. In the case without the top layer, the conversion efficiency $E_{\mathrm{c}}$ changes only slightly in a wide range of weight ratios, maintaining at about $20 \%$. By increasing the weight ratio above $w=30 \%$, the conversion efficiency $E_{\mathrm{c}}$ decreases as more light photons will bounce between phosphors and eventually dissipate into heats. In either case, the conversion efficiency $E_{\mathrm{c}}$ depends mildly on the phosphor size, but interestingly we still observe a conspicuous effect of size that smaller phosphors

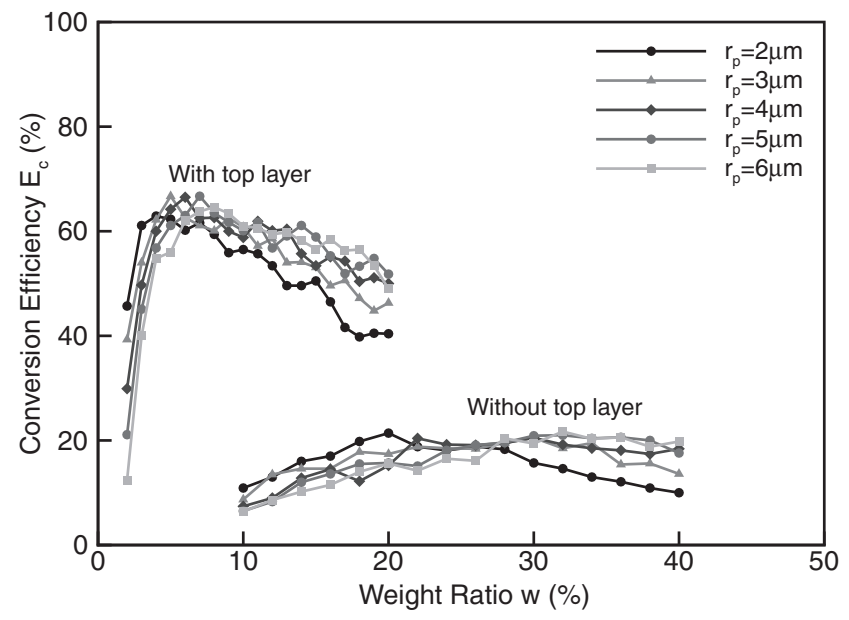

Fig. 5. The efficiency $E_{\mathrm{c}}$ of ultraviolet photons converted to visible photons for different weight ratios of phosphor particles with radius $r$ varying from 2 to $6 \mu \mathrm{m}$ with and without a top layer of omnidirectional photonic bandgap material.

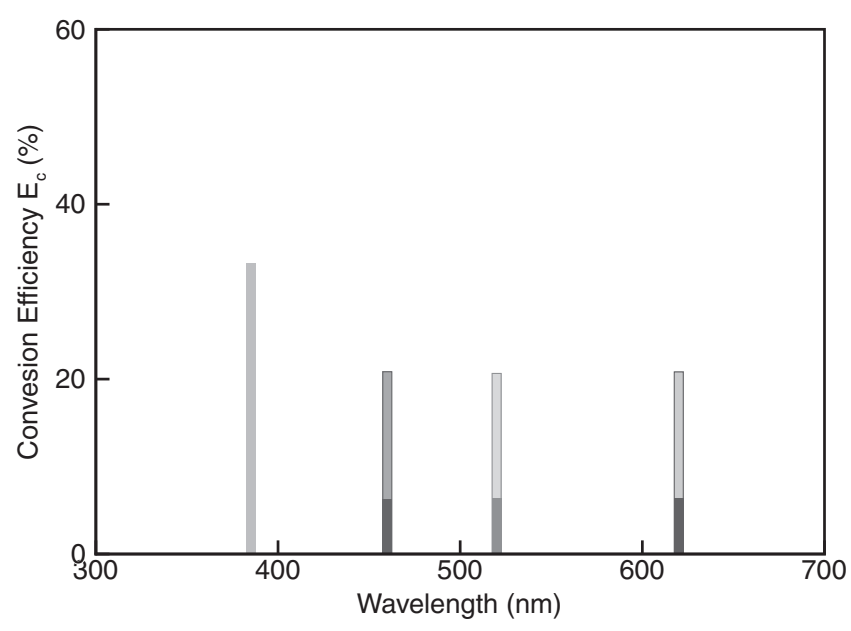

Fig. 6. Maximum percentages of red, green and blue photons converted from ultraviolet photons with (light, scaled from the bottom) and without (heavy) a top layer of omnidirectional photonic bandgap material.

are more efficient in conversion at lower weight ratios (relative to that at maximum conversion), while larger phosphors are more efficient at larger weight ratios.

Figure 6 shows the maximum percentages of red, green and blue photons converted from ultraviolet photons with and without top-covered omnidirectional photonic crystal for phosphors of size $3 \mu \mathrm{m}$. For a comparison, the line at $382 \mathrm{~nm}$ denotes the percentage of ultraviolet photons that escape the chamber in the case without the top layer. These light photons can be reused if a top layer of omnidirectional photonic bandgap material is inserted. The maximum conversion efficiency $\left(E_{\mathrm{c}}\right)_{\max }$ with the top layer improves by more than $200 \%$ compared the that without the top layer. The remarkable improvement is attributable to the fact that the ultraviolet light, when reaching the top layer, are reflected back into the chamber and can be reused to emit useful visible light. The conversion efficiency can be readily translated in terms of the unit of luminous flux (lumen) ${ }^{14)}$ to denote the strength of luminescence.

Furthermore, we investigate the cases in which the side wall and the bottom substrate are non-perfectly reflecting. This effect can be taken into account by sampling a uniformly distributed random number over $[0,1]$ to determine whether a light photon, when hitting a wall, is reflected by the wall. Figure 7 shows the conversion efficiency $E_{\mathrm{c}}$ versus the reflectivity $R$ of the side wall and the bottom substrate for a fixed chamber radius $r=5 \mathrm{~mm}$ but with varying the chamber height from $h=0.5$ to $5 \mathrm{~mm}$. In all the instances, the conversion efficiency $E_{\mathrm{c}}$ increases with increasing the reflectivity. The increasing rate for the case with a top layer of photonic bandgap materials is more rapid than for the case without a top layer. This is certainly due to the single effect that the photons are reused more efficiently in the case with a top layer, considering that all the other conditions are identically the same.

At lower wall reflectivity $R$, the best conversion efficiency $E_{\mathrm{c}}$ is observed to reach at the chamber height $h=1 \mathrm{~mm}$. There are three effects that strike a good balance at this chamber height. The chamber height cannot be too small as the ultraviolet photons would not have been efficiently converted to visible light by the phosphors. On the other 


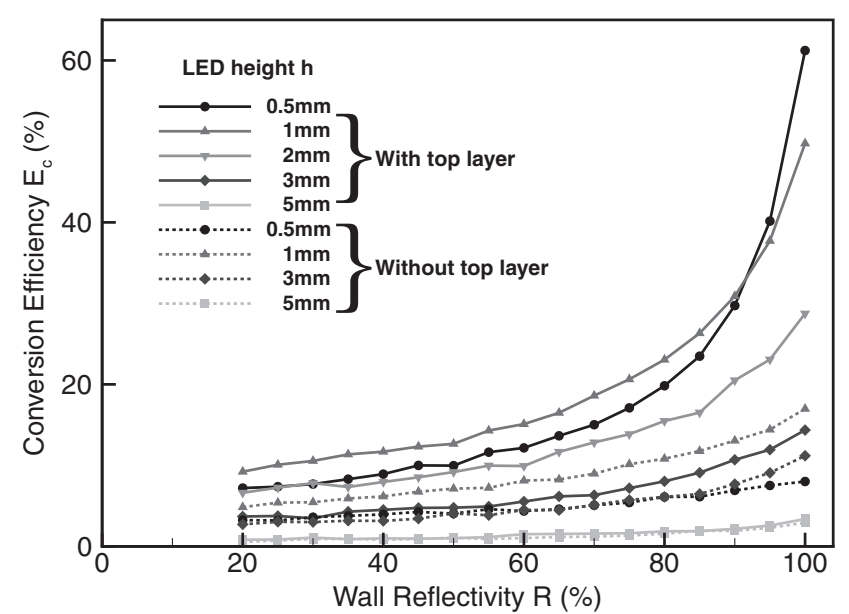

Fig. 7. The conversion efficiency $E_{\mathrm{c}}$ vs wall reflectivity for weight ratio $25 \%$ of phosphor particles with fixed chamber radius $5 \mathrm{~mm}$ and chamber heights from 0.5 to $5 \mathrm{~mm}$ with and without a top layer of omnidirectional photonic bandgap material.

hand, the chamber height $h$ cannot be too large as a large portion of photons would have been absorbed during their travelling in the chamber. Moreover, the high leakage of the side wall and bottom substrate at low reflectivity also tends to require a smaller chamber height. Consider the other extreme of perfectly reflecting side wall and bottom substrate. In the case with a top layer, the smallest chamber height $h=0.5 \mathrm{~mm}$ gives a very high conversion efficiency $E_{\mathrm{c}}=60 \%$ (compared to only $10 \%$ at the low wall reflectivity). However, the conversion efficiency $E_{\mathrm{c}}$ decreases rapidly with increasing the chamber height from $h=0.5$ to $5 \mathrm{~mm}$ as a result of the higher probability of absorption of a photon at a larger chamber height. At the larger chamber height, the conversion efficiency $E_{\mathrm{c}}$ increases only slightly from its value at low wall reflectivity, for most useful photons are lost in their travelling in the chamber. At about wall reflectivity $R=90 \%$, we observe a level crossing of the curves of conversion efficiency $E_{\mathrm{c}}$ for the chamber heights $h=0.5$ and $1.0 \mathrm{~mm}$. This indicates as the wall reflectivity is no longer an issue, the chamber with a top layer takes the small chamber height $h=0.5 \mathrm{~mm}$ for better conversion of ultraviolet photons.

In the case without a top layer of photonic bandgap materials, the best conversion efficiency $E_{\mathrm{c}}$ is also observed to reach at the chamber height $h=1.0 \mathrm{~mm}$. Even with a perfectly reflecting side wall, a smaller chamber height for less absorption does not overcome the leakage of useful photons out of the top substrate. The key observation is that none of these conversion efficiencies can be improved drastically by increasing the wall reflectivity. For example, the chamber with height $1.0 \mathrm{~mm}$ has the conversion efficiency $E_{\mathrm{c}}=15 \%$ for the case of perfectly reflecting walls compared to $5 \%$ at low wall reflectivity.

Figure 8 shows a different perspective of the effects of non-perfectly reflecting walls (side wall and bottom substrate) by fixing the chamber height. Again, we see the advantage by inserting a top layer of photonic bandgap materials which increases the conversion efficiency $E_{\mathrm{c}}$ less substantially at low wall reflectivity, but quite significantly at large wall reflectivity. In particular, for small chamber

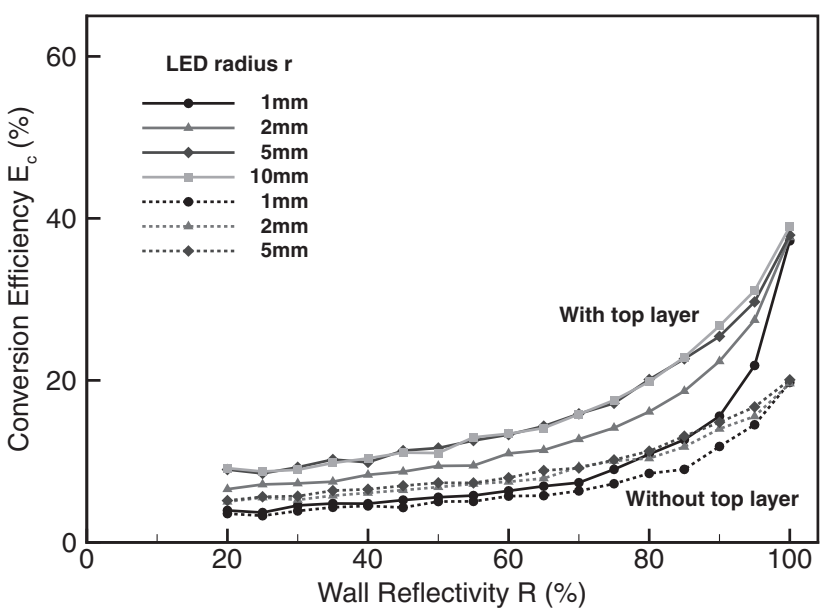

Fig. 8. The conversion efficiency $E_{\mathrm{c}}$ vs wall reflectivity for weight ratio $25 \%$ of phosphor particles with fixed chamber height $1.5 \mathrm{~mm}$ and chamber radii from 1 to $10 \mathrm{~mm}$ with and without a top layer of omnidirectional photonic bandgap material.

radius $r$ (say, $1 \mathrm{~mm}$ ), inserting the top layer of photonic bandgap materials does not improve at all the conversion efficiency in a wide range of lower wall reflectivity because the majority of photons leak out of the side wall and bottom substrate. As expected, the conversion efficiency at any wall reflectivity increases with increasing the chamber radius. However, we observe saturation of conversion at a certain chamber radius $r_{\mathrm{s}}$ (=3 $\mathrm{mm}$ in this case), above which the light-emitting device can no longer improve the conversion efficiency. The radius of saturation can be considered a index for the maximum activity region of the light photons as we have considered a point source of emission at the center of the bottom substrate, and thus it is of no use increasing further the chamber radius above it. It is further observed that as long as the side wall and the bottom substrate are perfectly reflecting, the conversion efficiencies $E_{\mathrm{c}}$ for different chamber radii converge to the same value (40\% for the cases with the top layer, and $18 \%$ for the cases without the top layer). Notice that there are two cases with radii (1 and $2 \mathrm{~mm}$ ) smaller than the radius of saturation $\left(r_{\mathrm{s}}=3 \mathrm{~mm}\right)$. This indicates that if the side wall and the bottom substrate are made perfectly reflecting, the lateral dimension of the chamber can thus be made smaller without loss of the conversion efficiency. However, this is not the case if the wall reflectivity $R$ deviates from 1 by a few percents, and at lower wall reflectivity it would be best to design the LED chamber at the radius of saturation $r_{\mathrm{s}}$ for a given chamber height $h$.

\section{Concluding Remarks}

In summary, we presented a very efficient Monte-Carlo method for simulating optical properties of a light-emitting chamber filled with randomly distributed phosphor particles. The present study indicates that it is crucial to use a top layer of omnidirectional photonic bandgap materials, with which the LED chamber can improve the conversion efficiency $E_{\mathrm{c}}$ drastically by increasing the wall reflectivity above $90 \%$, and at lower sidewall reflectivity, the chamber with the radius of saturation would be the most economic design. 


\section{Acknowledgments}

This work was supported in part by the Industrial Technology Research Institute under Contract No. 92S-23N0, and the National Science Council of the Republic of China under Contract No. NSC 91-2212-E-002-072, and the Ministry of Economic Affairs of the Republic of China under Contract No. MOEA 92-EC-17-A-08-S1-0006. The support from the National Center for Theoretical Sciences at Taipei is also acknowledged.

1) S. Nakamura, M. Senoh, S. Nagahama, N. Iwasa, T. Yamada, T. Matsushita, H. Kiyoku and Y. Sugimoto: Jpn. J. Appl. Phys. 35 (1996) L74.

2) P. Schlotter, J. Baur, C. Hielscher, M. Kunzer, H. Obloh, R. Schmidt and J. Schneider: Mater. Sci. Eng. B 59 (1999) 390.

3) T. Tamura, T. Setomoto and T. Taguchi: J. Lumin. 87 (2000) 1180.
4) J. K. Park, M. A. Lim, C. H. Kim, H. D. Park, J. T. Park and S. Y. Choi: Appl. Phys. Lett. 82 (2003) 683.

5) C. H. Kuo, J. K. Sheu, S. J. Chang, Y. K. Su, L. W. Wu, J. M. Tsai, C. H. Liu and R. K. Wu: Jpn. J. Appl. Phys. 42 (2003) 2284.

6) J. S. Kim, P. E. Jeon, Y. H. Park, J. C. Choi, H. L. Park, G. C. Kim and T. W. Kim: Appl. Phys. Lett. 85 (2004) 3696.

7) D. Xiao, K. W. Kim, S. M. Bedair and J. M. Zavada: Appl. Phys. Lett. 84 (2004) 672.

8) J. C. Su, J. R. Lo, C. H. Lin and J. Y. Chi: 5th Pacific Rim Conf. Lasers and Electro-Optics, Taipei, Taiwan, 2003, paper W2C.

9) Y. Fink, J. N. Winn, S. Fan, C. Chen, J. Michel, J. D. Joannopoulos and E. L. Thomas: Science 282 (1998) 1679.

10) K. Urabe: Jpn. J. Appl. Phys. 19 (1980) 885.

11) A. Konrad, J. Almanstotter, J. Reichardt, A. Gahn, R. Tidecks and K. Samwer: J. Appl. Phys. 85 (1999) 1796.

12) Y. Sato and S. Sato: Jpn. J. Appl. Phys. 39 (2000) 6281

13) K. N. Liou: An Introduction to Atmospheric Radiation (Academic Press, London, 2002).

14) S. Castelletto and M. L. Rastello: IEEE Trans. Instrum. Meas. 47 (1998) 900. 